\title{
Análisis geométrico y gráfico de las pirámides de Le Corbusier (1950-1957)
}

\author{
Geometric and graphical analysis of the pyramids of Le Corbusier (1950-1957) \\ Mariolly Dávila Cordido \\ Odart Graterol Prado \\ Universidad Simón Bolívar, Venezuela \\ Universidad Simón Bolívar, Venezuela \\ mariollydavila@usb.ve \\ odartgp@gmail.com
}

- José Javier Alayón González

Universidad Simón Bolívar, Venezuela

jalayon@usb.ve

\begin{abstract}
This paper addresses the issue about the geometrical properties and physical measures of the pyramids that Le Corbusier planned throughout his career; while approaching these bodies through his drawings and perspectives. This aims to decipher the objective role of mathematics, and the subjectivity of the visual-perceptive in his approach to the design process. Le Corbusier, one of the greatest masters of modern architecture, still uses the classical perspective as a tool that allows him to express the pictorial nature of his compositions and to demonstrate his aspirations about shape.
\end{abstract}

Keywords: Le Corbusier, Pirámide, Geometría, Perspectiva, Análisis

\section{Introducción}

Este trabajo analiza la geometría de las pirámides que Le Corbusier proyectó durante su carrera: las pirámides del estanque de la Capilla de Ronchamp (1950-55), la capilla mortuoria para los Delgado Chalbaud (1951) en Caracas, la pirámide del oratorio de los novicios de la Tourette (1953), también en Francia, el lucernario piramidal en Chandigarh, India (1955) y la pirámide de la cubierta del Museo de Tokio, Japón (1957, a través de la representación gráfica y virtual, para determinar el rol que juegan las proporciones en su arquitectura y el papel de la perspectiva en su proceso de proyecto.

Las proporciones de estos volúmenes las rigen los principios matemáticos y geométricos que Le Corbusier estudió y desarrolló en sus investigaciones y que se resumen en la escala del Modulor. Por ello, lo que nos ocupa es que la forma y la proporción de estos casos de estudio -desde los determinados puntos de vista-, se ajustan a ellos y producen efectos ópticos controlados, y no azarosos.

Le Corbusier era capaz de prever esas ilusiones ópticas y corregirlas para lograr una aprehensión de la forma, una apariencia específica, que varía en todos los casos que estudiaremos, determinando similitudes y diferencias entre ellas a partir de su comparación detallada y de la comprobación geométrica.

Esta aproximación a una de las formas puras, citada por el arquitecto suizo y representada en su "Lección de Roma" por la pirámide de Cayo Cestius, busca explicar lo que el propio Le Corbusier exponía en 1923: "La fuerza de intención, la clasificación de los elementos, es prueba de un cambio de espíritu: estrategia, legislación. La arquitectura es sensible a estas intenciones, produce. La luz acaricia las formas puras: esto produce. Los volúmenes simples desarrollan inmensas superficies que se enuncian con una variedad característica según se trate de cúpulas, bóvedas, cilindros, prismas rectangulares o pirámides" (Le Corbusier, 1998, p. 128-127)

Por ejemplo, la proporción y la forma del volumen de la pirámide de la capilla mortuoria encargada como tumba para el General Delgado Chalbaud y su padre en Caracas, está proyectada para conseguir un efecto visual contrario a la deformación que por su base triangular refleja. Cuando se dibuja en el sistema diédrico se percibe una repetición y simetría del triángulo escaleno que se completa en el sistema cónico. Esto ayuda a ubicar los elementos arquitectónicos en puntos estratégicos como el acceso o el tratamiento de sus superficies, para evitar la deformación descontrolada de la pirámide y volver a observarlo como en los planos frontal y lateral de la doble ortogonal. O como juega con los materiales y la materialidad del muro permeable o, simplemente, la ubicación de las ventanas para la búsqueda de una espacialidad interior específica a través de la luz que penetra de manera controlada. 


\section{Procedimientos metodológicos}

A partir de estas observaciones, el estudio de estas pirámides es un ejercicio del tipo multidisciplinar en el que forman parte, los referentes históricos, geométricos y tecnológicos para el desarrollo de su proceso proyectual. La metodología se puede definir en cuatro fases, de las cuales la primera y la segunda son comunes, mientras que la tercera y la cuarta son específicas para el dibujo.

\section{Primera fase}

Levantamiento detallado de las parcelas o emplazamientos ocupados por las pirámides estudiadas, la mayoría de ellas integradas en perímetros ortogonales y bien definidos.

\section{Segunda fase}

Documentación y recogida de datos. Rastreo de fuentes documentales. Investigación bibliográfica y documental que incluye la comprobación de las fuentes útiles para la reconstrucción virtual, y que luego se incluirán en la memoria explicativa del proyecto.

\section{Tercera fase}

Comparación geométrica y de dibujo en doble ortogonal y en perspectiva de las pirámides en cuestión. En paralelo se analizan su obra artística, dibujos y perspectivas en donde aparecen referencias triangulares e incluso piramidales y su particular de la perspectiva como herramienta de diseño.

\section{Cuarta fase}

Comprobación de las proporciones e intenciones proyectuales del arquitecto a partir de los modelos de pirámides deducidas de estas referencias y los parámetros de diseño, geométricos, constructivos y el empleo de los sistemas de representación gráfica; diédrico y cónico.

Esta metodología se podría ampliar a otros elementos y/o edificios de interés de la obra de Le Corbusier y de la arquitectura moderna, en donde la geometría juega un papel importante.

Para hacer una reconstrucción virtual arquitectónica, como en el caso elegido de Caracas, con una base científica, es necesario un conocimiento profundo de la arquitectura de Le Corbusier y su idea de arquitectura durante la década del 50 del s. XX. La construcción histórica constituye un aspecto clave en la tecnología infográfica para poder garantizar la cientificidad del producto.

En el análisis de estas pirámides subyace el interés por comprender el proceso proyectual de Le Corbusier a partir del estudio de la geometría como algo objetivo y contrastando sus representaciones gráficas, principalmente perspectivas, cargadas de subjetividad y manipulación.

\section{La perspectiva como dibujo objetivo}

La perspectiva cónica frontal, es aquella donde el espacio converge en líneas en un punto de fuga sobre la línea de horizonte. Este tipo de representación nos ofrece información geométrica de las proporciones de los elementos que contienen la escena en sus verdaderas magnitudes. La perspectiva queda definida o enmarcada por la disposición del objeto, que generalmente está en paralelo con el plano de encuadre, y su base debe estar apoyada o en paralelo al plano horizontal o plano del piso. Debido a su espacialidad acotada entre el punto de vista del observador y el plano de proyección, una vez hecha la perspectiva, podemos determinar las dimensiones de los elementos de dos maneras: a través de las dimensiones reales que están contenidas en alguno de los planos o mediante cálculos a partir del espacio. Por ello, este tipo de perspectiva ha sido recurrente a lo largo de la historia de la arquitectura, esté o no cargada de subjetividad.

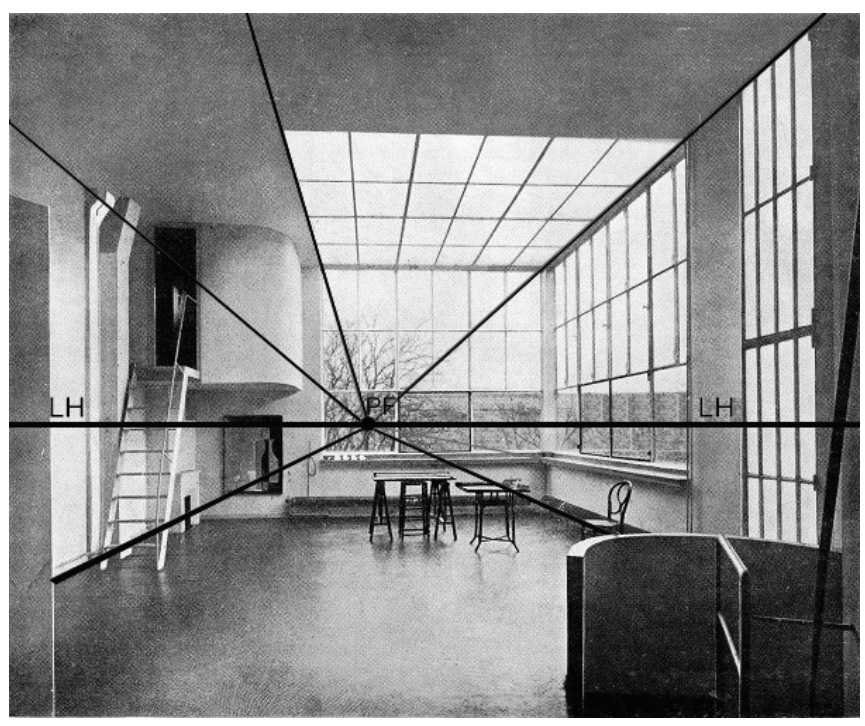

Figura 1. Interior de la casa-taller del pintor Amédée Ozenfant. Fotografía de Le Corbusier.

A partir de este tipo de representación espacial, en otras ocasiones (Dávila, 2011), hemos podido revertir el proceso que contiene la información gráfica de plantas y alzados, como en el caso del Monumento funerario Delgado Chaulbaud en donde se obtuvo información complementaria para reconstruir tridimensionalmente lo que era imposible leer en el resto de información planimetríca. Cuando se establece solo un punto de fuga, y el plano de encuadre se encuentra paralelo al plano de proyección, la representación con profundidad limitada- de ese espacio, sigue permitiendo la comprensión y evaluación de las relaciones geométricas de la composición, aproximándola a una representación planimétrica. 


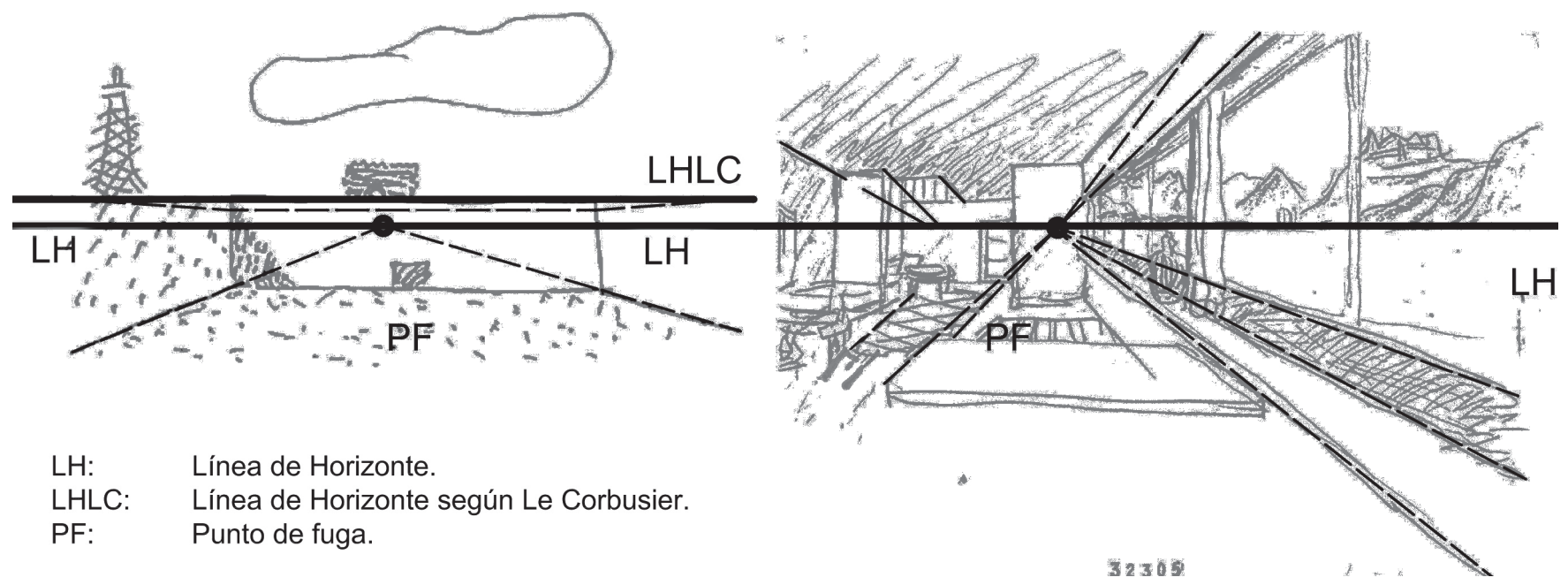

Figura 2: Ejemplos de perspectivas con punto de fuga (PF) único. En el caso del Ático de París, la línea superior del muro se hace coincidir con una falsa línea de horizonte, mientras que el dibujo de la villa "Le Lac" respeta fielmente las líneas de fuga.

\section{Las perspectivas de Le Corbusier, dibujos subjetivos}

En algún momento, Le Corbusier afirmó que el plano y la sección contenían todo el proyecto, sentencia que denostaba la perspectiva como método de representación, un método que Bruno Zevi llegó a calificar de "venenoso" y castrador de la educación espacial.

Esta minusvaloración de la perspectiva, y en general de la visualidad, llegó al punto de afectar la propedéutica del arquitecto a partir de la modernidad. Sin embargo, la mayoría de los arquitectos modernos nunca abandonó este método como parte de las herramientas de comprobación del proyecto, eso sí, desvinculada ya de su papel en la historia de la pintura, pues las vanguardias -en especial la revolución cubista- se encargaron de desterrarla de su ámbito, para cederla a la arquitectura como instrumento aséptico de demostración de lo que vemos, una simple representación de la realidad.

No obstante, Le Corbusier no termina de vaciar a la perspectiva de su contenido sensitivo, y la utiliza como método de transmisión de su pensamiento. Sus perspectivas, como las que vemos en la figura 3, pueden llegar a manipular conscientemente algunos aspectos espaciales o formales que quiere potenciar. En estos casos específicos, la duplicidad de puntos de fuga dilata horizontalmente el espacio.

Esta configuración clásica de un solo punto de fuga centrado, históricamente ligada a la perspectiva lineal del Renacimiento y su concepción espacial, evidencia la constante contradicción o combinación de conceptos clásicos y modernos en el proyecto lecorbuseriano. Ese centro simbólico que la modernidad rechazaba, permanece anclado en muchas perspectivas del arquitecto suizo, - véase el caso de la pirámide de Caracas-, como punto de referencia que sin duda lo relaciona con la perspectiva premedieval que Erwin Panofsky describe, en donde los elementos se disponen como un bodegón dentro de una composición, con apenas profundidad.

Las pirámides que estudiamos, son figuras dinámicas, resultantes de bases triangulares irregulares cuya cúspide busca un centro, o por el contrario, por el desplazamiento de esta cúspide hacia alguno de los bordes, cuando la planta es una forma regular cuadrada como en los casos de La Tourette y Chandigarh. Con ello, Le Corbusier deforma la figura platónica en función de su comportamiento dentro del campo que ocupa. Estos recintos son los que condicionan realmente la lectura de las pirámides por el modo en que nos aproximamos y desplazamos.

Las perspectivas en los bocetos iniciales de Le Corbusier poseen un particular carácter pictórico. En ellas puede apreciarse la preferencia por el uso de un solo punto de fuga en lugar de los dos o tres comúnmente usados por otros maestros modernos. Parece haber una intención preconcebida en la manera de proyectar el espacio y los objetos como son representados dentro del mismo. Apela a un aplanamiento de la perspectiva tridimensional en una muestra de predilección por la composición bidimensional de los objetos. Así la representación de un "espacio sistemático" como lo define E. Panofsky en su libro "La perspectiva como forma simbólica" pasa a un segundo plano, anteponiendo a la veracidad representativa la relación existente entre los objetos que componen la obra. Panofsky nos dice:

"El espacio representado se convierte en un espacio de agregados y no en el espacio que la época moderna exige y realiza (...) Porque lo que designamos como tendencia moderna presupone siempre una unidad superior más alládel espacio vacío de los cuerpos, de modo que desde un principio la contemplación de ellos, observados a través de estos presupuestos, conserve su orientación y unidad; y por esto nunca puede, a través de una tan extendida depreciación y disolución de la forma estable, poner en peligro la estabilidad de la imagen espacial y la compacidad de las diferentes cosas, sino que solo puede velarla; mientras que la antigüedad, careciendo de esta unidad superior, sólo a costa de una disminución del orden de la corporeidad, puede lograr un aumento en el orden de la espacialidad, de tal forma que el espacio parece alimentarse de las cosas y para las cosas 


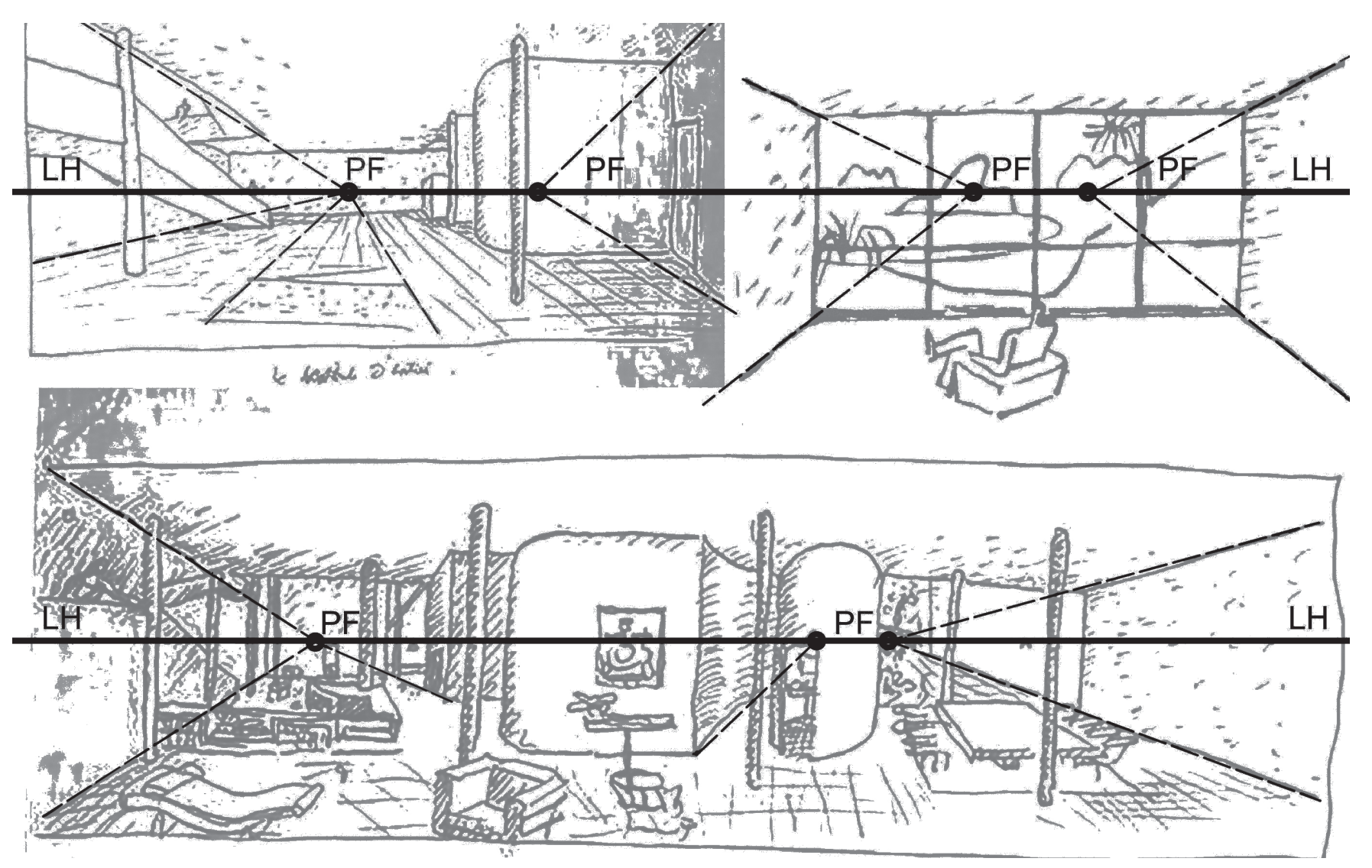

Figura 3: Dibujos de Le Corbusier en donde se duplican o triplican los puntos de fuga.
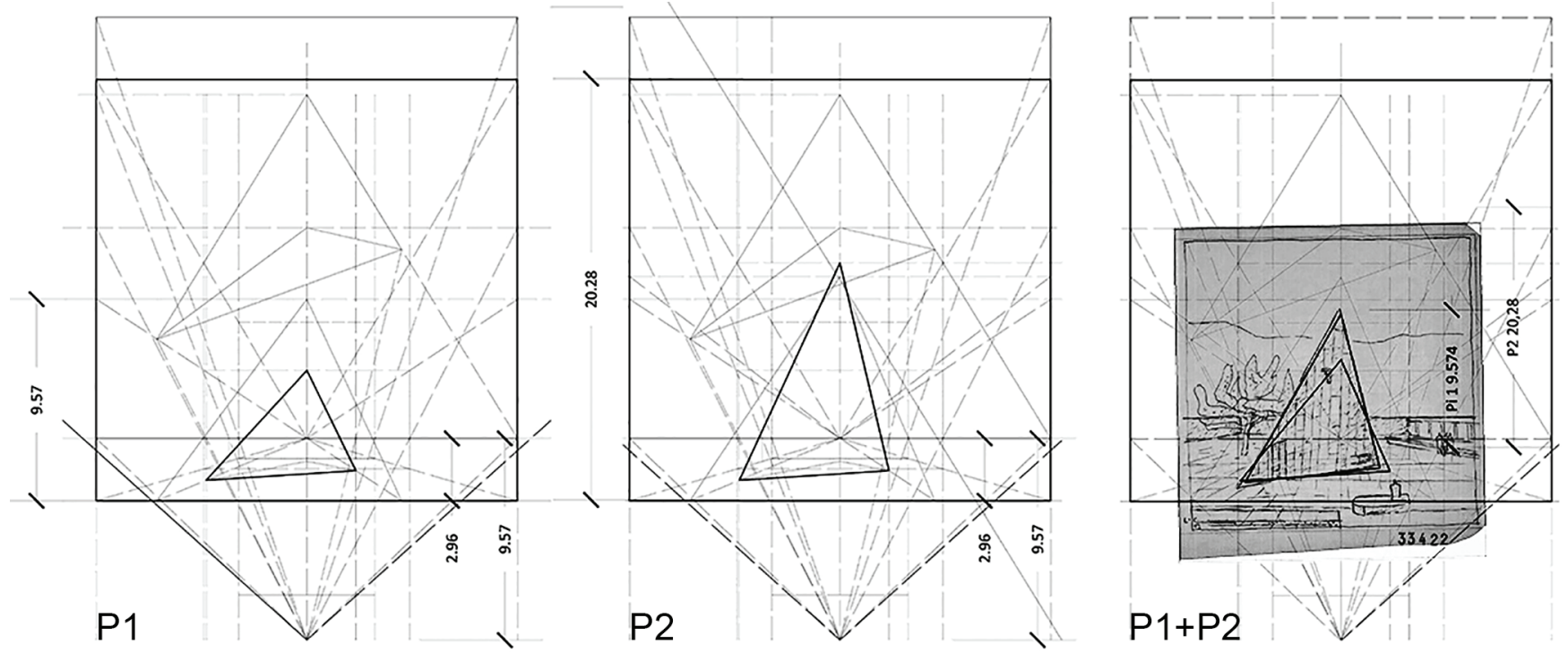

Figura 4: P1.Reconstrucción de la perspectiva según Le Corbusier con los valores de las proporciones que nos brindan los alzados. Altura del observador: $2.95 \mathrm{~m}$. Distancia: 9,57 m. Altura según alzados: 9,57 m. P2. Reconstrucción de la perspectiva según le Corbusier, con las proporciones que nos brinda la misma perspectiva. Altura del observador: $2.95 \mathrm{~m}$. Distancia: 9,57 m. Altura de la pirámide según alzados: $20,28 \mathrm{~ms}$. P1+P2. Superposición de P1 y P2 sobre dibujo original de Le Corbusier CFLC-ADAGP Altura del observador: 2.95 m. Distancia: 9,57 m. Altura según alzados: 9,57 y $20.28 \mathrm{~ms}$. 
y justamente esto explica el fenómeno, paradójico hasta ahora, de que el mundo del arte antiguo, siempre que renunciemos a la representación del espacio intercorporal, resulta más sólido y armónico que el moderno..." (Panofsky, 1995. pp.25)

A pesar de la preocupación del arquitecto por la sistematización explicitada principalmente en un sistema de proporciones (El Modulor), que al proyectar podríamos circunscribir dentro de un espacio teórico, adscrito a leyes matemáticas; existe una preocupación aun mayor, en términos de representación inicial, por un espacio estético, y que no son sino traducciones de una misma sensibilidad del espacio perceptivo, que en uno y otro caso aparecen o bien sometido a leyes lógicas como en el primer caso o bien simbolizado, como en el segundo. Le Corbusier toma siempre partido por éste último, no solo en las representaciones iniciales de proyectos, bocetos y dibujos, sino también en la disposición del encuadre para las tomas fotográficas. (Fig.1)

\section{La pirámide de Caracas en perspectiva, entre lo} objetivo y lo subjetivo

Para explicar con detalle la relación entre los aspectos geométricos (objetivos) y los puramente visuales (subjetivos) seleccionamos el Monumento Funerario para el General Delgado Chalbaud y su padre, por ser la única de las pirámides no construidas y uno de los proyectos menos conocidos de Le Corbusier.

Para reconstruir con herramientas informáticas la perspectiva que hizo Le Corbusier a mano alzada se utilizaron los mismos puntos de vista y la misma distancia a fin de recrear la escena y las proporciones de la pirámide, es decir; un observador a 2.95 metros de altura y una distancia al plano de encuadre de 9,57 metros. En el cuadro P1, observamos la pirámide de 9,57 metros de altura, muy diferente en proporciones a la que encontramos en la perspectiva hecha por Le Corbusier. En el cuadro P2, se vuelve a dibujar la perspectiva con la proporción de la pirámide que le Corbusier dibuja en la perspectiva y que sugiere ser de 20,28 metros, una de las medidas de la serie roja de su Modulor, y por último en el cuadro P1+P2, superponemos las dos perspectivas técnicas sobre el dibujo original que hace Le Corbusier.

De este ejercicio se deduce que existe una gran diferencia entre la concepción geométrica objetiva del cuerpo piramidal, basado en su sistema de proporciones y la concepción visual, subjetiva, de la percepción de la pirámide. Esta manipulación de la perspectiva demuestra que, en primera instancia, el arquitecto dibuja lo que quiere, no lo que ve.

\section{Conclusiones geométricas y gráficas}

Comparando las pirámides entre sí, nos encontramos con que su emplazamiento está girado en el plano que las
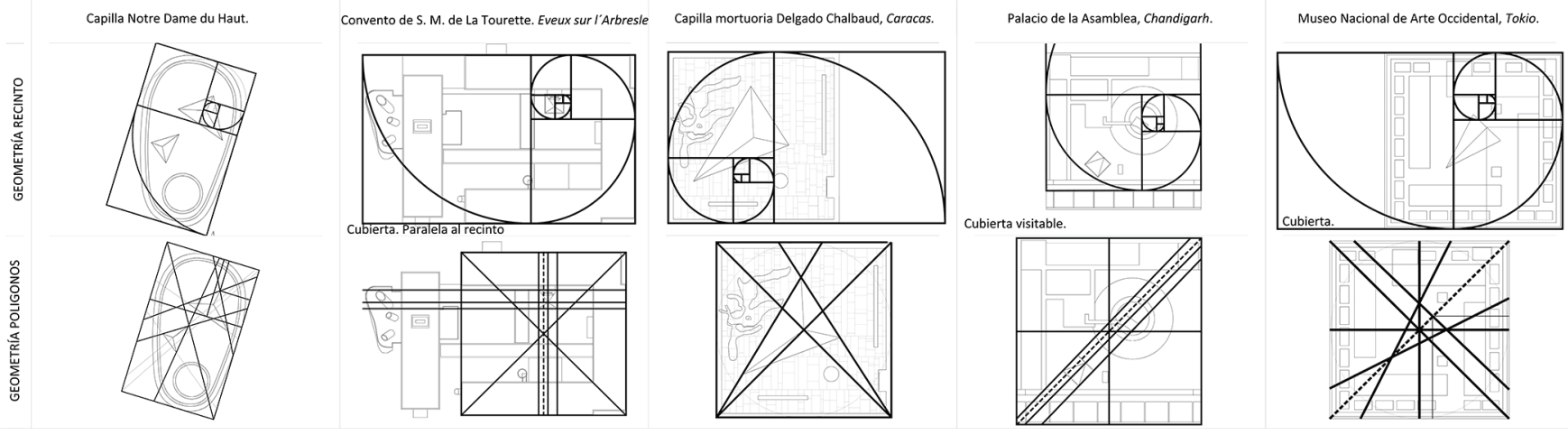

Figura 5: Relaciones geométricas de las pirámides con relación a sus recintos.
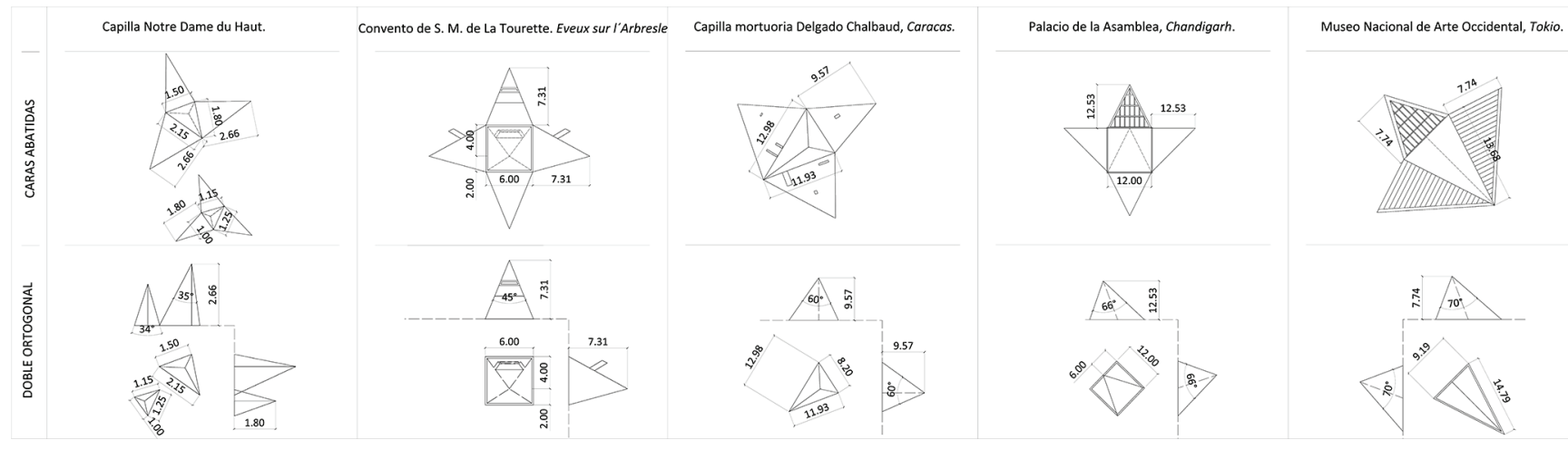

Figura 6: Caras abatidas de las pirámides y sus dimensiones. 
contiene, salvo en el monasterio de La Tourette, en donde el volumen piramidal de base cuadrada se encuentra paralelo a las paredes del claustro. En los casos de Chandigarh y Tokio, las pirámides poseen una simetría axial, en donde todos los semiplanos tomados a partir de dicho eje y conteniéndolo presentan idénticas características. No obstante, su disposición dentro del recinto, como ya dijimos, no corresponde con una concepción lineal del espacio.

Solo en el caso del monumento funerario de Caracas, donde la pirámide es protagonista absoluta del conjunto, su forma es el resultado de unas trazas reguladoras claras que lo unen a su recinto de una manera indisociable. Estas líneas reguladoras certifican el sistema de proporciones creado por Le Corbusier y están al servicio de una búsqueda armónica de la forma. En este caso particular, la perspectiva oblicua frontal que traza desde un punto elevado y fuera del recinto, sirve a Le Corbusier para la comprobación de las proporciones, las cuales, dentro de esta visión subjetiva, responden a una lógica que nada tiene que ver con lo propuesto en las fachadas o secciones definitivas del proyecto.

En el resto de casos, la geometría juega un papel menos determinante. Recordemos que allí, las pirámides son parte de unos conjuntos arquitectónicos más amplios y complejos.

Salvo las pirámides de la Tourette y del Palacio de la Asamblea en Chandigarh, cuyas bases son cuadradas, en el resto son irregulares. Sin embargo, en ninguna, la cúspide coincide con el centro geométrico de la base. Progresivamente, desde Ronchamp hasta Tokio, las cúspides se van acercando a una de las caras, hasta llegar a ser coplanares. En ese caso, las caras que son perpendiculares al plano de apoyo son lucernarios. Estás pirámides son las más regulares y por ello menos dinámicas del grupo estudiado. En estas pirámides, la forma sigue a la función.

En cualquier caso, Le Corbusier rehúye de la figura platónica, forzándola a responder o dialogar con una geometría invisible de trazados reguladores o con una función específica y con su entorno a través de una respuesta ambiental.

Otra particularidad geométrica de las pirámides, es la relación entre alto y ancho, siendo las primeras muy esbeltas para luego ir reduciendo su altura en relación a la base que define la circunferencia donde se circunscribe. Es importante aclarar, que no todas son habitables, y que la base de algunas "flotan" sobre el espacio, no coincidiendo su base con el plano habitable.

Esta doble aproximación a las pirámides lecorbuserianas, nohace sinocorroborar el planteamientodualqueLeCorbusier amalgama en su obra: objetivo-subjetivo, naturaleza-artificio, razón-pasión.

\section{Referencias}

Dávila, M. (2011). Estudio para la valoración y recuperación del patrimonio arquitectónico religioso Venezolano a través de técnicas digitales: iglesia de San Jacinto, caso de estudio (Tesis Docotral). Universitat Politècnica de Catalunya. Escola Técnica Superior d'Arquitectura de Barcelona, Departament d'Expressió Gràfica Arquitectònica. Barcelona,España. http:// hdl.handle.net/10803/52804

Lapunzina, Alejandro (2002). La Pirámide y el Muro: notas preliminares sobre una obra inédita de Le Corbusier en Venezuela. Massilia: anuario de estudios lecorbusierianos, $\mathrm{N}^{\circ}$. 2002, 148-161.

Le Corbusier (1998) Hacia una arquitectura (1ª reimpr. de la $2^{\text {a }}$ ed.). Barcelona: Ediciones Apóstrofe.

Le Corbusier (1980). El Modulor 2. Barcelona: Poseidón.

Le Corbusier et Pierre Jeanneret (1964) CEuvre complète 19101929. Zurich: Les Editions d'architecture.

Panofsky, Erwin (1995). La perspectiva como forma simbólica (7 $7^{\text {a }}$ edición), Barcelona: Tusquets Editores. 